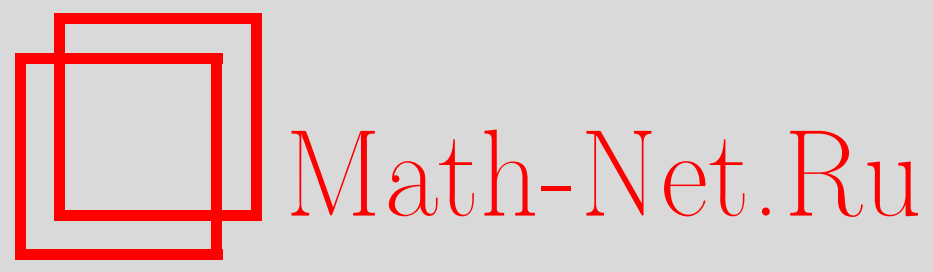

В. И. Сердобольский, Асимптотически доминирующее оценивание векторов математических ожиданий, Теория вероятн. и ее примен., 1999, том 44, выпуск $1,132-138$

DOI: https://doi.org/10.4213/tvp606

Использование Общероссийского математического портала Math-Net.Ru подразумевает, что вы прочитали и согласны с пользовательским соглашением

http://www.mathnet.ru/rus/agreement

Параметры загрузки:

IP : 18.209 .158 .208

26 апреля 2023 г., 17:24:05

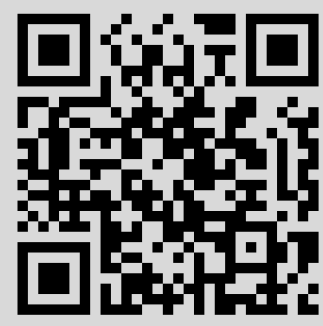


где

$$
|\omega| \leqslant 2 \Delta \sqrt{2 \pi}+\frac{\psi(x)}{\lambda x} A_{3}<2 \sqrt{2 \pi} \Delta+\frac{A_{3}}{\rho(\varepsilon)}=A_{4} .
$$

Легко усмотреть, что функция $A_{4}=A_{4}(\varepsilon)$ возрастает в интервале $\left[0, \rho^{-1}\left(\frac{1}{4}\right)\right)($ см. $(17)-(19),(27)$ и $(30))$. Поэтому в теореме в качестве $\tilde{\theta}(r)$ можно выбрать функцию $A_{4}\left(\rho^{-1}(r)\right), 0 \leqslant r \leqslant \frac{1}{4}$.

Итак,

$$
\tilde{\theta}(r)=\left.A_{4}(\varepsilon)\right|_{\varepsilon=\rho^{-1}(r)},
$$

$$
A_{4}(\varepsilon)=1.583 \sqrt{2 \pi}\left(1+\varepsilon+\frac{1}{2} \varepsilon^{2}+\frac{1}{3} \varepsilon^{3}+\frac{11}{24} \frac{\varepsilon^{4}}{1-\tau \varepsilon}\right)(1-A)^{-1 / 2}+\varepsilon \frac{\varepsilon}{\rho(\varepsilon)} \frac{1}{\sqrt{1-A}} A_{2}
$$

где $A=A(\varepsilon), A_{2}=A_{2}(\varepsilon)$ и $\rho(\varepsilon)$ определены в (18), (27) и (28) соответственно.

Заметим, что оценку для $\theta$ в (3) при умеренных $x$ можно несколько уточнить: из (31) и (32) следует, что $|\theta| \leqslant 2 \sqrt{2 \pi} \Delta+\psi(x) A_{3} / \rho(\varepsilon)$.

Для завершения доказательства теоремы воспользуемся (31), равенством $-\frac{x^{2}}{2} Q(x)=V-h \bar{\alpha}+\frac{1}{2} x^{2}$ (см. [2, (46), (47), (51), (52)]), а также результатами работы $[5,(3),(7),(8)]$, в которой найдены явные формулы и оценки (4) для коэффициентов $q_{\nu}$.

\section{СПИСОК ЛИТЕРАТУРЫ}

1. Feller $W$. Generalization of a probability limit theorem of Cramer. - Trans. Amer. Math. Soc., 1943, v. 54, № 3, p. 361-372.

2. Lenart C. On certain theorems of Berry and a limit theorem of Feller. - Matematcý časopis, 1968 , v. 18 , № 1 , p. 59-75.

3. Розовский Л. В. Одно обобщение теоремы Колмогорова о законе повторного логарифма уклонений. - Теория вероятн. и ее примен., 1997, т. 42, в. 1, с. 134-143.

4. Архангельский A.H. Нижние оценки для вероятностей больших уклонений сумм независимых случайных величин. - Теория вероятн. и ее примен., 1989, т. XXXIV, в. 4 , с. $625-635$.

5. Розовский Л. В. О коэффициентах ряда Крамера. - Теория вероятн. и ее примен., 1998, т. 43 , в. 1 , с. $161-166$.

6. Шиганов И. С. Об уточнении верхней константы в остаточном члене центральной предельной теоремы. Проблемы устойчивости стохастических моделей. Труды семинара. М.: ВНИИСИ, 1982, с. 109-115.

Поступила в редакцию

12.III.1998

(C) 1999 г. $\quad$ СЕРДОБОЛЬСКИЙ В. И.*

\section{АСКМПTОTИЧЕСКИ ДОМИНИРУЮШЕE ОЩЕНИВАНИЕ ВEКTOPOB МАТЕМАТИЧЕСКИХ ОЖИДАНИЙ}

Решается задача минимизации квадратичного риска покомпонентно компрессионных оценок математических ожиданий нормальных векторов с независимыми компонентами с единичной дисперсией. Найдена наилучшая компрессионная функция, и построена компрессионная оценка вектора математических ожиданий, наилучшая с точностью до членов, малых при большой размерности и большом объеме выборки.

* Московский государственный институт электроники и математики, Б. Трехсвятительский пер., 3/12, 109028 Москва, Россия; e-mail: serd@miem.edu.ru 
Ключевые слова и фразы: компрессионные оценки, доминируюшие оценки, большая размерность.

В 1956 г. Стейн обнаружил [1], что в многомерном случае выборочное среднее может не обладать наименьшим квадратичным риском. Известно, что оценки векторов математических ожиданий вида $\eta \bar{x}$, где $\overline{\mathrm{x}}$ есть $n$-мерный вектор средних выборочных, а $\eta<1$ («компрессионные оценки») при $n>2$ могут обладать меньшим квадратичным риском для нормальных распределений и некоторых других центрально симметричных распределений [2], [3]. В предлагаемой работе эффект компрессии оценок изучается в рамках подхода асимптотики растущей размерности (a.p.p., см. [4]), когда объем выборки сколь угодно велик, а отношение размерности наблюдений к объему выборки ограничено. Так же, как в [5], будем исследовать эффект покомпонентного компрессионного оценивания и искать оценки, асимптотически неулучшаемые в а.p.p. Но в отличие от [5] будем анализировать главные члены а.p.p., не переходя к пределу, оценивая остаточные члены с точностью до абсолютных констант.

Обозначим $\mathrm{x}=\left(x_{1}, \ldots, x_{n}\right)$ вектор наблюдений, $\mu=\left(\mu_{1}, \ldots, \mu_{n}\right)=\mathbf{E x}-$ вектор параметров. Предположим, что $\mathrm{x} \sim \mathbf{N}(\mu, \mathbf{I})$, где $\mathbf{I}$ - единичная матрица. Пусть $\overline{\mathbf{x}}=$ $\left(\bar{x}_{1}, \ldots, \bar{x}_{n}\right)$ - вектор средних, рассчитанный по выборке объема $N$. Квадратичный риск оценки $\overline{\mathrm{x}}$, очевидно, равен $n / N$.

Утверждение 1. Оченка Стейна $\widehat{\mu}^{S}=\left(1-(n-2) / N \overline{\mathrm{x}}^{2}\right) \overline{\mathrm{x}}($ здесь и ниже квадраты векторов означают квадраты их длия) при $n>2$ обладает следуючим приблиэсенно экстремальным свойством:

$$
\mathbb{E}\left(\mu-\widehat{\mu}^{S}\right)^{2} \leqslant \min _{\eta} \mathbb{E}(\mu-\eta \overline{\mathbf{x}})^{2}+\frac{4(n-1)}{n N},
$$

где $\eta$ - неслучайный скаляр. Здесь экстремальное значение $\eta$ равно $\mu^{2}\left(\mu^{2}+n / N\right)^{-1}$.

Исследуем эффект покомпонентного оценивания вектора математических ожи-

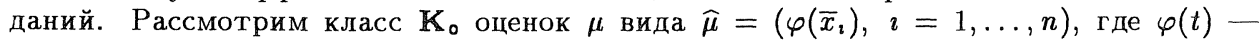
неслучайная измеримая функция с ограниченным ростом: $|\varphi(t)| \leqslant c|t|$, а $c$ от $t$ не зависит. Будем минимизировать $\mathrm{E}(\boldsymbol{\mu}-\widehat{\mu})^{2}$. Воспользуемся основным приемом a.p.p.: рассматривать множества величин в виде функций их эмпирического распределения. Обозначим

$$
\mathscr{F}_{0}(t)=n^{-1} \sum_{\imath} \text { ind }\left(\bar{x}_{\imath} \leqslant t\right), \quad F_{0}(t)=\mathbb{E} \mathscr{F}_{0}(t)=n^{-1} \sum_{\imath} \Phi\left(\sqrt{N}\left(t-\mu_{\imath}\right)\right)
$$

(здесь и ниже в суммах $\imath=1, \ldots, n)$. Обозначим

$$
f_{0}(t)=\frac{d F_{0}(t)}{d t}=n^{-1} \sqrt{\frac{N}{2 \pi}} \sum_{i} \exp \left(-\frac{N\left(t-\mu_{i}\right)^{2}}{2}\right) .
$$

Утверждение 2. Функция $\varphi(t)=\varphi^{0}(t)=t+N^{-1} \partial \ln f_{0}(t) / \partial t$ обеспечивает минимальный квадратичный риск оченок из $\mathrm{K}_{\mathrm{o}}$, причем

$$
\inf \mathbb{E}(\widehat{\mu}-\mu)^{2}=\frac{n}{N}-\frac{n}{N^{2}} \int\left(\frac{\partial}{\partial t} \ln f_{\mathrm{o}}(t)\right)^{2} f_{\mathrm{o}}(t) d t .
$$

В частном случае, когда $\mu_{1}=\mu_{2}=\cdots=\mu_{n}$, квадратичный риск $R^{\circ}=0$.

При оценивании $f_{0}(t)$ по $\mathscr{F}_{\circ}(t)$ необходимо сглаживание. Для удобства выделения главных членов изменим масштаб и перейдем к равносильной задаче оценивания векторов $\mathrm{v}=\left(v_{1}, \ldots, v_{n}\right)$ параметров $v_{\imath}=\sqrt{n} \mu_{\imath}$ по статистике $\mathrm{u}=\left(u_{1}, \ldots, u_{n}\right)$, где $u_{\imath}=\sqrt{n} \bar{x}_{\imath}, \imath=1, \ldots, n$. Рассмотрим класс $\mathbb{K}$ оценок $\mathrm{v}$ вида

$$
\widehat{\mathrm{v}}=\left(\varphi\left(u_{\imath}\right), \imath=1, \ldots, n\right),
$$

где по-прежнему $|\varphi(t)| \leqslant c|t|$ для всех $t$ и $c$ от $t$ не зависит. Обозначим $y=n / N$, $R(\varphi)=n^{-1} \mathrm{E}(\widehat{\mathrm{v}}-\mathrm{v})^{2}, f(t, d)=n^{-1} 1 / \sqrt{2 \pi d} \sum_{\imath} \exp \left(\left(t-v_{\imath}\right) /(2 d)\right), d>0$. 
Утверждение 3. Справедливы соотношения

$$
\begin{aligned}
& R(\varphi)=R_{\mathrm{opt}}+\int\left[\varphi(t)-\varphi^{\circ}(t, y)\right]^{2} f(t, y) d t \\
& R_{\mathrm{opt}}=R\left(\varphi^{\circ}\right)=y-y^{2} \int\left[\frac{\partial}{\partial t} \ln f(t, y)\right]^{2} f(t, y) d t, \quad \varphi^{\circ}(t, y)=t+y \frac{\partial}{\partial t} \ln f(t, y) .
\end{aligned}
$$

Таким образом, оценка

$$
\mathrm{v}^{\circ}=\left(\varphi^{\circ}\left(u_{1}, y\right), \ldots, \varphi^{\circ}\left(u_{n}, y\right)\right)
$$

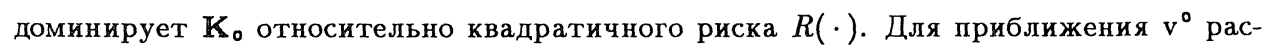
смотрим статистику

$$
\hat{f}(t)=\hat{f}(t, \varepsilon)=n^{-1} \sum_{i}(2 \pi \varepsilon)^{-1 / 2} \exp \left(-\frac{\left(t-u_{i}\right)^{2}}{2 \varepsilon}\right), \quad \varepsilon>0 .
$$

Лемма 1. При $\varepsilon>0$

$$
\mathbf{E} \hat{f}(t)=f(t, y+\varepsilon), \quad \operatorname{var} \hat{f}(t) \leqslant n^{-1}(2 \pi \varepsilon)^{-1} f(t, d), \quad \text { zде } d=y+\frac{\varepsilon}{2} .
$$

Чтобы построить оценку $\varphi^{\circ}(t, y)$ с ограниченным ростом, необходимо ограничить убывание $\hat{f}(t, \varepsilon)$ при больших $t$. Рассмотрим статистики

$$
\begin{aligned}
\widehat{\varphi}^{\circ}(t, \varepsilon, \delta) & =t+\operatorname{ind}(\hat{f}(t)>\delta) y \frac{\partial}{\partial t} \ln \hat{f}(t), \quad \varepsilon, \delta>0, \\
\widehat{\mathrm{v}}^{\circ} & =\left(\widehat{\varphi}\left(u_{1}, \varepsilon, \delta\right), \ldots, \widehat{\varphi}\left(u_{n}, \varepsilon, \delta\right)\right) .
\end{aligned}
$$

Теорема 1. Можно указать такие функиии $\varepsilon=\varepsilon(n, N)<y$ и $\delta=\delta(n, N)$, например, $\varepsilon=y n^{-18 / 143}$ u $\delta=y^{-1 / 2} n^{-40 / 143}$, что при любых натуральных $n$ и $N$

$$
R\left(\widehat{\mathrm{v}}^{0}\right) \leqslant\left(\sqrt{R_{\mathrm{opt}}}+\sqrt{\rho}\right)^{2}
$$

где $0<\rho<a у n^{-1 / 4}$, и а-абсолютная константа.

Следствие 1. Оченка (3) с $\varepsilon=\varepsilon(n, N)$ u $\delta=\delta(n, N)$ из теоремы 1 обладает следуюиим асимптотически доминируючим свойством: каковы бы ни были с >0 $u \omega>0$, можно указать такое $n_{0}=n_{0}(c, \omega)$, что при $n_{0}<n<c N$ для любых $\widehat{\mathrm{v}} \in \mathrm{K}$ $R\left(\widehat{\mathrm{v}}^{\circ}\right) \leqslant R(\widehat{\mathrm{v}})+\omega$.

Относительный квадратичный риск оценки $\mathrm{v}^{0}$ с наилучшей неизвестной оценивающей функцией $\varphi^{\circ}(t)$ равен $r^{0}=R\left(\varphi^{\circ}\right) / y<1$. Относительный риск оценки (3) составляет не больше $\left(\sqrt{r_{\text {opt }}}+a n^{-1 / 4}\right)^{2}$, где $a-$ абсолютная константа. В этом смысле оценки $\widehat{\mathrm{v}}^{0}$ при $n \rightarrow \infty$ асимптотически доминируют класс оценок $\mathbf{K}$, причем равномерно как по совокупностям, так и по объемам выборок.

Отметим, что при больших $n$ и $N$ предположение о нормальности может быть заменено на предположение об асимптотической нормальности. Можно ожидать [6], что при ограниченной зависимости переменных теорема 1 может быть установлена для широкого класса совокупностей.

Доказательства. Замечаем, что для любой ограниченной функции $\psi(t)$ $\sum_{i} \mathbf{E} \psi\left(u_{i}\right)=\int \psi(t) f(t, d) d t$. Чтобы доказать лемму 1, достаточно проверить несмещенность оценки интегрированием и заметить, что дисперсия $\hat{f}(t, \varepsilon)$ составляет

$$
\begin{gathered}
n^{-2}(2 \pi \varepsilon)^{-1} \sum_{i} \operatorname{var} \exp \left(-\frac{\left(t-u_{i}\right)^{2}}{2 \varepsilon}\right) \leqslant n^{-2}(2 \pi \varepsilon)^{-1} \sum_{i} \operatorname{E} \exp \left(-\frac{\left(t-u_{i}\right)^{2}}{\varepsilon}\right) \\
=n^{-2}(4 \pi \varepsilon)^{-1 / 2}(2 \pi d)^{-1 / 2} \sum_{i} \exp \left(-\frac{\left(t-v_{i}\right)^{2}}{2 d}\right), \quad d=y+\frac{\varepsilon}{2}, \varepsilon>0 .
\end{gathered}
$$

Докажем, что квадратичный риск оценки (3) векторов v при больших $n$ и некоторых $\varepsilon$ и $\delta$ приближается к квадратичному риску $R_{\text {opt }}$ оценки (2). Для этого оценим 
увеличение квадратичного риска при переходе от $\varphi^{\circ}(t, y)$ к $\varphi^{\circ}(t, y+\varepsilon)$, а затем от $\varphi^{\circ}(t, y+\varepsilon)$ к $\widehat{\varphi}^{\circ}(t, \varepsilon, \delta)$. Обозначим

$$
\begin{aligned}
& \rho_{1}=\operatorname{En}^{-1} \sum_{i}\left[v_{\imath}-\varphi^{\circ}\left(u_{\imath}, y\right)\right]^{2}, \quad \rho_{2}=\operatorname{En}^{-1} \sum_{i}\left[\varphi^{\circ}\left(u_{i}, y\right)-\varphi^{\circ}\left(u_{i}, y+\varepsilon\right)\right]^{2} \\
& \rho_{3}=\operatorname{En}^{-1} \sum_{i}\left[\varphi^{\circ}\left(u_{i}, y+\varepsilon\right)-\widehat{\varphi}^{\circ}\left(u_{\imath}, \varepsilon, \delta\right)\right]^{2}
\end{aligned}
$$

Доказательство основной теоремы опирается на следующие леммы.

Лемма 2. Пусть $\varepsilon>0$. Величина $\rho_{2} \leqslant a \varepsilon^{2} / y$, где $a-$-абсолютная константа. Д ок азат ель с т в о. Обозначим для краткости $x_{\mathfrak{\imath}}=\left|v_{\mathfrak{\imath}}-t\right| / d, d>0$,

$$
f_{\imath}=\frac{1}{\sqrt{2 \pi d}} \exp \left(-\frac{\left(t-v_{\imath}\right)^{2}}{2 d}\right), \quad f_{\imath t}=\frac{\partial}{\partial t} f_{\imath}, \quad f_{\imath d}=\frac{\partial}{\partial d} f_{\imath}, \quad f_{\imath t d}=\frac{\partial^{2}}{\partial d \partial t} f_{\imath} .
$$

Выразим $\rho_{2}$ через производные функции $\varphi^{0}$, взятые при промежуточном значении $d_{i}: y \leqslant d_{\imath} \leqslant y+\varepsilon$. Находим, что

$$
\rho_{2}=n^{-1} \varepsilon^{2} \mathrm{E} \sum_{\imath}\left[\frac{\partial \varphi^{0}\left(u_{\imath}, d\right)}{\partial d}\right]^{2}=n^{-1} \varepsilon^{2} \mathrm{E} \sum_{\imath}\left(\frac{\left|f_{\imath t}\right|}{f_{\imath}}+\frac{\left|f_{\imath t d}\right|}{f_{\imath}} d_{\imath}+\frac{\left|f_{\imath t} f_{\imath d}\right|}{f_{\imath}^{2}} d_{\imath}\right)^{2},
$$

где под знаком суммы $d=d_{\imath}=d_{\imath}\left(u_{\imath}\right), \imath=1, \ldots, n$. Вычисление математического ожидания при каждом $\imath$ сводится к интегрированию по $u_{\imath}$ с весом $(2 \pi y)^{-1 / 2} \exp \left(-\left(u_{\imath}-\right.\right.$ $\left.\left.v_{\imath}\right)^{2} / 2 y\right) \leqslant \sqrt{d_{\imath} / y} f_{\imath}$. Оставляя квадраты слагаемых, получаем

$$
\rho_{2} \leqslant 3 n^{-1} \varepsilon^{2} \mathbf{E} \sum_{\imath} \sqrt{\frac{d_{\imath}}{y}}\left(\frac{f_{\imath t}^{2}}{f_{\imath}}+\frac{f_{\imath t d}^{2}}{f_{\imath}} d_{\imath}^{2}+\frac{f_{\imath t}^{2} f_{\imath d}^{2}}{f_{\imath}^{3}} d_{\imath}^{2}\right),
$$

где для каждого $\imath$ переменные $d=d_{\imath}$.

Обозначим $x_{\imath}=\left(v_{\imath}-u_{\imath}\right) / \sqrt{d_{\imath}}, \imath=1, \ldots, n$. Находим, что $f_{\mathfrak{t}}^{2} \leqslant x_{\imath}^{2} f_{\imath}^{2} / d_{\imath} \leqslant\left(x_{\imath}^{2}+\right.$ $1)^{2} f_{\imath}^{2} / 4 d_{\imath}, f_{\imath t d}^{2} \leqslant\left(x_{\imath}^{2}+2\left|x_{\imath}\right|+1\right)^{2} f_{\imath}^{2} / 4 d_{\imath}^{3}$. Поэтому

$$
\rho_{2} \leqslant a \varepsilon^{2} \mathbf{E} n^{-1} \sum_{\imath} \frac{1}{\sqrt{d_{\imath} y}}\left(x_{\imath}^{2}+x_{\imath}^{6}\right) f_{\imath} \leqslant b \varepsilon^{2} y^{-3 / 2} n^{-1} \sum_{\imath} \mathbf{E}\left(x_{\imath}^{2}+x_{\imath}^{6}\right) \exp \left(-\frac{x_{\imath}^{2}}{2}\right) .
$$

Здесь $a$ и $b-$ абсолютные константы. Каждый член под знаком $\mathbf{E}$ в последней оценке сверху не больше константы. Заключаем, что $\rho_{2} \leqslant a \varepsilon^{2} y^{-1}$. Лемма доказана.

Величину $\rho_{3}$ разобьем на два слагаемых

$$
\begin{aligned}
& \rho_{31}=\mathbf{E} n^{-1} \sum_{\imath}\left[\varphi^{\circ}\left(u_{\imath}, y+\varepsilon\right)-\widehat{\varphi}^{0}\left(u_{\imath}, \varepsilon, \delta\right)\right]^{2} \text { ind }\left(\hat{f}\left(u_{\imath}\right)>\delta\right), \\
& \rho_{32}=d^{2} n^{-1} \mathbf{E} \sum_{\imath} r^{2}\left(u_{\imath}\right) \text { ind }\left(\hat{f}\left(u_{\imath}\right) \leqslant \delta\right),
\end{aligned}
$$

где $r(t)=r(t, d)=\partial \ln f(t, d) / \partial t, d=y+\varepsilon$.

Лемма 3. При $0<\varepsilon \leqslant y u n>1$

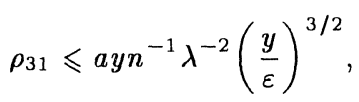

где $\lambda=\delta \sqrt{2 \pi y}$ иа-абсолютная константа.

Доказ а т ел в с т в о. Обозначим

$$
\begin{aligned}
\hat{f}_{\imath}(t) & =(2 \pi \varepsilon)^{-1 / 2} \exp \left(-\frac{\left(t-u_{\imath}\right)^{2}}{2 \varepsilon}\right), \\
\xi_{\imath}(t) & =r(t, d) \hat{f}_{\imath}(t)-\frac{d}{d t} \hat{f}_{\imath}(t)=\hat{f}_{\imath}(t)\left[r(t, d)+\frac{t-u_{\imath}}{\varepsilon}\right], \\
q(t) & =n^{-1} \sum_{\imath} \xi_{\imath}(t), \quad \imath=1, \ldots, n .
\end{aligned}
$$


Находим, что

$$
\rho_{31} \leqslant y^{2} \delta^{-2} \mathbb{E} n^{-1} \sum_{\imath}\left[r\left(u_{\imath}, d\right) \hat{f}\left(u_{\imath}\right)-\hat{f}^{\prime}\left(u_{\imath}\right)\right]^{2} \text { ind }\left(\hat{f}^{\imath}\left(u_{\imath}\right)>\delta\right) \leqslant y^{2} \delta^{-2} n^{-1} \sum_{\imath} \mathbb{E} q^{2}\left(u_{\imath}\right),
$$

здесь и ниже штрих означает производную по явно выписанному аргументу. Для любого неслучайного $t$ имеем $\mathbb{E} q(t)=r(t, d) f(t, d)-\partial f(t, d) / \partial t=0$. Следовательно, $\mathbf{E} q^{2}(t)=\operatorname{var} q(t)=n^{-2} \sum_{\imath} \operatorname{var} \xi_{2}(t)$. Величина

$$
\begin{aligned}
\mathbf{E} \hat{f}_{\imath}^{2}(t) & =(2 \pi \varepsilon)^{-1}(2 \pi y)^{-1 / 2} \int \exp \left[-\frac{(t-z)^{2}}{\varepsilon}-\frac{\left(z-v_{\imath}\right)^{2}}{2 y}\right] d z \\
& =(2 \pi)^{-1} \varepsilon^{-1 / 2} \tilde{d}^{-1 / 2} \exp \left(-\frac{\left(t-v_{\imath}\right)^{2}}{2 \widetilde{d}}\right), \quad \imath=1, \ldots, n
\end{aligned}
$$

где $\tilde{d}=y+\varepsilon / 2$. Значит, при любых $t$ и є эта величина не больше $(2 \pi)^{-1 / 2}(y \varepsilon)^{-1 / 2}$. Величина

$$
\begin{aligned}
\mathbb{E}\left[f_{\imath}^{\prime}(t)\right]^{2} & =(2 \pi \varepsilon)^{-1} \int(2 \pi y)^{-1 / 2} \exp \left[-\frac{(t-z)^{2}}{\varepsilon}-\frac{\left(z-v_{\imath}\right)^{2}}{2 y}\right] \frac{(t-z)^{2}}{\varepsilon^{2}} d z \\
& \leqslant(4 \pi \sqrt{2})^{-1} y^{-1 / 2} \varepsilon^{-3 / 2}, \quad \imath=1, \ldots, n .
\end{aligned}
$$

Находим, что при любом $t: \mathbb{E} \xi^{2}(t)<\pi^{-1} \varepsilon^{-1 / 2} y^{-1 / 2}\left[r^{2}(t, d)+\varepsilon^{-1}\right], \jmath=1, \ldots, n, d=$ $y+\varepsilon, \varepsilon>0$. Выделим зависимость от $u_{\imath}$ : пусть $\mathbf{E}=\mathbb{E}_{\imath} \mathbb{E}^{\imath}$, где $\mathbb{E}_{\imath}$ вычисляется интегрированием только по распределению величины $u_{i}$, а $\mathbb{E}^{i}-$ по распределению остальных $u_{\jmath}, \jmath \neq \imath$. Очевидно,

$$
\begin{aligned}
\mathbb{E}^{2} q(t) & =n^{-1}\left(\xi_{\imath}(t)-\mathbb{E}_{\imath} \xi_{\imath}(t)\right), \\
\mathbb{E}^{2} q^{2}(t) & =n^{-2}\left[\sum_{\jmath \neq \imath} \operatorname{var} \xi_{\jmath}(t)+\left(\xi_{\jmath}(t)-\mathbb{E}_{\imath} \xi_{\jmath}(t)\right)\right], \quad \imath=1, \ldots, n .
\end{aligned}
$$

Докажем, что второе слагаемое в квадратных скобках дает лишь поправку к первому. Замечаем, что $\xi_{\imath}\left(u_{\imath}\right)=(2 \pi \varepsilon)^{-1 / 2} r\left(u_{\imath}, d\right)$, а при неслучайном аргументе $t$

$$
\left|\mathbb{E}_{\imath} \xi_{z}(t)\right|=(2 \pi d)^{-1 / 2} \exp \left(-\frac{\left(t-v_{\imath}\right)^{2}}{2 d}\right)\left|r(t, d)+\frac{t-v_{\imath}}{d}\right| \leqslant \frac{r(t, d)+d^{-1 / 2}}{\sqrt{2 \pi d}},
$$

$\imath=1, \ldots, n$. Получаем оценку $\mathbb{E}^{\imath} q^{2}\left(u_{\imath}\right) \leqslant n^{-2} s\left(u_{\imath}\right), \imath=1, \ldots, n$, где $s(t)=$ $\sum_{\jmath=1}^{n} \operatorname{var} \xi_{\jmath}(t)+r^{2}(t, d) / \varepsilon+d^{-2}$. Находим, что

$$
n^{-1} \sum_{\imath} \mathbb{E} q^{2}\left(u_{\imath}\right)=n^{-1} \sum_{\imath} \mathbb{E}_{\imath}\left[\mathbb{E}^{2} q(t)\right]_{t=u_{\imath}} \leqslant n^{-2} \int s(t) f(t, y) d t .
$$

Подставляем оценку сверху для $\operatorname{var} \xi_{\jmath}(t)$ через второй момент и пользуемся утверждением 3 (при $y=d$ ). Находим, что (при $\varepsilon<y$ ) правая часть не больше $\pi^{-1} n^{-1} \varepsilon^{-3 / 2} y^{-1 / 2}\left(1+\theta+2 \pi \theta^{1 / 2} n^{-1}\right)$, где $\theta=\varepsilon / y$. Получаем утверждение леммы.

Пемма 4. При $0<\varepsilon \leqslant y$ для пюбого положительного $\alpha<1$

$$
\frac{\rho_{32}}{2} \leqslant y n^{-1} \lambda^{-2}\left(\frac{y}{\varepsilon}\right)^{1 / 2}+a(1-\alpha)^{-3 / 2} y \lambda^{\alpha}
$$

где $\lambda=\delta \sqrt{2 \pi y}, a-$ абсолютная константа.

Д ок аз а т ел в с т в о. Обозначим

$$
\hat{f}^{\imath}(t)=n^{-1}(2 \pi \varepsilon)^{-1 / 2}\left[\sum_{\jmath \neq \imath} \exp \left(-\frac{\left(t-u_{\jmath}\right)^{2}}{2 \varepsilon}\right)+1\right], \quad \imath=1, \ldots, n
$$

Находим, что

$$
\begin{aligned}
\frac{\rho_{32}}{2} & \leqslant y^{2} \mathbb{E} n^{-1} \sum_{i} r^{2}\left(u_{\imath}, d\right) \text { ind }\left(\hat{f}^{1}\left(u_{\imath}\right) \leqslant \delta\right)=y^{2} n^{-1} \sum_{i} \mathbb{E}_{\imath} r^{2}\left(u_{\imath}, d\right) \mathbb{P}\left(\hat{f}^{2}\left(u_{\imath}\right) \leqslant \delta\right) \\
& \leqslant y^{2} n^{-1} \sum_{i} \mathbb{E}_{\imath} r^{2}\left(u_{\imath}, d\right)[\mathbb{P}(\hat{f}(t) \leqslant \delta)]_{t=u_{\imath}}=y^{2} \int r^{2}(t, d) \mathbb{P}(\hat{f}(t) \leqslant \delta) f(t, y) d t
\end{aligned}
$$


где $d=y+\varepsilon, \varepsilon>0$. Здесь $f(t, y) \leqslant d^{1 / 2} y^{-1 / 2} f(t, d)$, и правая часть оценки $\rho_{32}$ не превосходит $\int y^{3 / 2} d^{1 / 2} r^{2}(t, d) \mathbf{P}(\hat{f}(t) \leqslant \delta) f(t, d) d t, d=y+\varepsilon, \varepsilon>0$. Разбиваем этот интеграл на два интеграла $I_{1}$ и $I_{2}$ по областям $\mathbf{S}_{1}=\{t: f(t, d) \geqslant 2 \delta\}$ и $\mathbf{S}_{2}=$ $\{t: f(t, d)<2 \delta\}, \rho_{32} \leqslant I_{1}+I_{2}$. В $\mathbf{S}_{1}$ согласно неравенству Чебышева

$$
\mathbf{P}(\hat{f}(t)<\delta) \leqslant \mathbf{P}\left[\frac{|\hat{f}(t)-\mathbf{E} \hat{f}(t)|}{\sigma}>\frac{\delta}{\sigma}\right] \leqslant \frac{\sigma^{2}}{\delta^{2}},
$$

где $\sigma^{2}=\operatorname{var} \hat{f}(t) \leqslant n^{-1}\left(8 \pi^{2} \varepsilon y\right)^{-1 / 2}$. При $\delta=\lambda / \sqrt{2 \pi d}$ отношение $\sigma^{2} / \delta^{2}<$ $n^{-1}(y / \varepsilon)^{1 / 2} \lambda^{-2}$. В силу утверждения $3 \int r^{2}(t, d) f(t, d) d t \leqslant 1 / d$ и, значит, $I_{1} \leqslant$ $y n^{-1}(y / \varepsilon)^{1 / 2} \lambda^{-2}$.

Оценим теперь $I_{2}$. Обозначим для краткости $e_{i}=\exp \left(-\left(t-v_{i}\right)^{2} / 2 d\right)$ и будем записывать средние по $i=1, \ldots, n$ угловыми скобками. Например, $f(t, d)=(2 \pi d)^{-1 / 2}\left\langle e_{i}\right\rangle$. Находим, что

$$
I_{2}=\left(\frac{y}{d}\right)^{3 / 2} \int_{\mathbf{S}_{2}} \frac{\left\langle e_{i}\left(t-v_{i}\right)\right\rangle^{2}}{\left\langle e_{i}\right\rangle} \mathbf{P}(\hat{f}(t)<\delta) f(t, d) d t \leqslant\left(\frac{y}{d}\right)^{3 / 2}(2 \pi d)^{-1 / 2} \int_{\mathbf{S}_{2}}\left\langle e_{i}\left(t-v_{i}\right)^{2}\right\rangle d t .
$$

Пусть $0<\alpha<1, \beta=(1-\alpha) / 2$. Произведение $e_{i}^{\beta}\left(t-v_{i}\right)^{2} \leqslant d / \beta$ при любом $i$, откуда находим, что

$$
I_{2} \leqslant(2 \pi)^{-1 / 2} y^{3 / 2} \beta^{-1} d^{-1} \int_{\mathbf{S}_{2}}\left\langle e_{i}^{\alpha+\beta}\right\rangle d t .
$$

Обозначим $l=\max _{i}\left|v_{i}\right|, \psi(t)=\pi^{-1} l\left(l^{2}+t^{2}\right)^{-1}$. Интеграл в правой части последнего неравенства не больше

$$
\int_{\mathbf{S}_{2}} \sqrt{\left\langle e_{i}^{2 \alpha}\right\rangle\left\langle e_{i}^{2 \beta}\right\rangle} d t \leqslant \lambda^{\alpha} \int \sqrt{\left\langle e_{i}^{2 \beta}\right\rangle} d t \leqslant \lambda^{\alpha}\left[\int \frac{\left\langle e_{i}^{2 \beta}\right\rangle}{\psi(t)} d t\right]^{1 / 2} .
$$

Последний интеграл

$$
\int\left\langle e_{i}^{2 \beta}\right\rangle \pi l^{-1}\left(l^{2}+t^{2}\right) d t=\pi^{3 / 2} \frac{d}{\beta}\left(2 l+\frac{d}{2 l \beta}\right) \leqslant 2 \pi^{3 / 2} \frac{d}{\beta} .
$$

Получаем оценку $I_{2}<2^{3 / 2} \pi^{1 / 4} \lambda^{\alpha}(1-\alpha)^{-3 / 2} y$. Лемма доказана.

Док а за те льст в о те о ре мы 1. Опираемся на леммы 2,3 и 4 . Легко убедиться, что

$$
R\left(\widehat{\mathrm{v}}^{\circ}\right)=\mathbf{E} n^{-1}\left(\widehat{\mathrm{v}}^{0}-\mathrm{v}\right)^{2} \leqslant R_{\mathrm{opt}}+2 \sqrt{R_{\mathrm{opt}}}\left(\sqrt{\rho_{2}}+\sqrt{\rho_{31}}+\sqrt{\rho_{32}}\right)+5 \rho_{2}+2 \rho_{31}+2 \rho_{32} .
$$

Положим, например, $\alpha=0.9$. Замечаем, что первое слагаемое в оценке величины $\rho_{32}$ согласно леммам 3 и 4 при $\varepsilon \leqslant y$ не больше оценки $\rho_{31}$. Приравниваем $\rho_{2}, \rho_{31}$ и второе слагаемое $\rho_{32}$ (без коэффициентов). Находим, что $y \theta^{2}=n^{-1} \delta^{-2} \theta^{-3 / 2}=y^{1.45} \delta^{0.9}$, где, как и выше, $\theta=\varepsilon / y$. Эти уравнения удовлетворяются при $\theta=y n^{-18 / 143}, \delta \sqrt{y}=$ $n^{-40 / 143}$. При этом $\rho=y n^{-36 / 143} \leqslant y n^{-1 / 4}$. Теорема доказана.

\section{СПИСОК ЛИТЕРАТУРЫ}

1. Stein C. M. Inadmissibility of the usual estimator for the mean of a multivariate normal distribution. - In: Proceedings of the Third Berkeley Symposium on Mathematical Statistics and Probability, 1956, v. 1, p. 197-206

2. Стейн Ч. Лекции по теории оценивания многих параметров. - Записки ЛОМИ, 1977, т. 74, c. $4-65$.

3. Shao P. Y., Strawderman W.E. Improving on the James-Stein positive-part estimator. - Ann. Statist., 1994, v. 42, p. 1517-1538.

4. Айвазян С.А., Бухштабер В.М., Енюков И. С., Мешалкин Л.Д. Прикладная статистика. М.: Финансы и статистика, 1989. 
5. Сердобольский В. И. Асимптотически доминирующее оценивание векторов математических ожиданий растущей размерности. - В сб.: Случайный анализ. М.: МГУ, 1987 , с. 87-96.

6. Сердобольский В. И. Нормализация функционалов качества многомерных статистических процедур. - Докл. РАН, 1995, т. 343, с. 743-745.

Поступила в редакцию

23.I.1992

Переработанный вариант

1.VII.1997

1999 г.

ЧУПРУНОВ А. Н.*

\title{
О СХОДИМОСТИ ПО РАСПРЕДЕЛЕНИЮ МАКСИМУМОВ НЕЗАВИСИМЫХ ОДИНАКОВО РАСПРЕДЕЛЕННЫХ СЛУЧАЙНЫХ ВЕЛИЧИН СО СЛУЧАЙНЫМИ КОЭФФИЦИЕНТАМИ ${ }^{1)}$
}

\begin{abstract}
В статье приведены предельные теоремы для максимумов независимых одинаково распределенных случайных величин, принадлежаших области нормального притяжения макс-устойчивой случайной величины со случайными коэффициентами, обладаюшими свойством малости на бесконечности или его аналогами. Изучается сходимость случайных ступенчатых функций, определенных такими максимумами в пространстве Скорохода. Описываются предельные распределения.
\end{abstract}

Ключевые слова и фразы: независимые одинаково распределенные случайные величины, сходимость по распределению, функциональная предельная теорема, принцип инвариантности, макс-устойчивые распределения.

1. Введение. Пусть даны: $\left\{k_{n}\right\}, n \in \mathbf{N},-$ последовательность натуральных чисел такая, что $k_{n} \rightarrow \infty, n \rightarrow \infty ; \xi_{n \imath}, 1 \leqslant \imath \leqslant k_{n}, n \in \mathbf{N}$, - последовательность серий независимых в каждой серии неотрицательных случайных величин; $\eta, \eta_{\mathbf{s}}, \imath \in \mathbf{N},-$ последовательность независимых одинаково распределенных случайных величин с общей функцией распределения $F$ таких, что $\left\{\xi_{n_{\imath}}\right\},\left\{\eta, \eta_{\imath}\right\}$ - независимые семейства. Введем последовательности случайных величин:

$$
M_{n}=\max _{1 \leqslant \imath \leqslant k_{n}} \xi_{n \mathfrak{r}} \eta_{1}, \quad M_{p n}=n^{-1 / p} \max _{1 \leqslant 1 \leqslant n} \eta_{1}, \quad M_{-p n}=n^{1 / p} \max _{1 \leqslant \imath \leqslant n} \eta_{\mathfrak{l}}, \quad n \in \mathbf{N},
$$

где $p>0$. Пусть $\zeta_{1 c p}$ и $\zeta_{2 c p}-$ случайные величины с функциями распределения соответственно, где $c>0$

$$
F_{1 c p}(x)=\left\{\begin{array}{ll}
\exp \left(-c x^{-p}\right), & x>0, \\
0, & x \leqslant 0,
\end{array} \quad \text { и } \quad F_{2 c p}(x)= \begin{cases}1, & x \geqslant 0 \\
\exp \left(-c(-x)^{p}\right), & x<0\end{cases}\right.
$$

Сходимость нормированных максимумов независимых одинаково распределенных случайных величин к случайным величинам $\zeta_{1 с p}$ и $\zeta_{2 c p}$ изучалась в [1] (см. также монографию $[2$, с. 50$])$. Эта заметка посвящена изучению сходимости последовательности $M_{n}$ (п. 2) и сходимости случайных ступенчатых функций, определенных случайными величинами $M_{n}$ (п. 3) при условии, что последовательность $M_{p n}$ сходится

* НИИММ им. Чеботарева, ул. Университетская, 17, 420008 Казань, Татарстан.

1) Работа выполнена при поддержке Российского фонда фундаментальных исследований, грант 96-01-01265. 